\title{
Precision asphere polishing with MRF technology and Subaperture Stitching Interferometry
}

\author{
Sławomir Gogler, 'Grzegorz Bieszczad, Michał Krupiński \\ Institute of Optoelectronics, Military University of Technology, 2 gen. S. Kaliskiego Str., Warsaw, Poland
}

Received October 15, 2013; accepted December 17, 2013; published December 31, 2013

\begin{abstract}
The paper presents the results of aspheric lens manufacturing in Infrared Technology and Thermovision Section in the Military University of Technology. Briefly MRF and SSI technologies utilized in the process are described and a case study of three aspherization processes follows. The first two elements are silicon lenses designed for a mid-wave infrared (MWIR) lens. The third one is a parabolic mirror substrate. Technical aspects and requirements of each case are discussed.
\end{abstract}

Aspheric lens manufacturing always posed a challenge. Not only is it difficult to manufacture aspheric shape but also it is difficult to measure it. Since it is impossible to manufacture something that cannot be measured the problem is two-fold. Even though aspheric lens shapes are as old as the law of refraction itself until recently their use has been relatively limited. With the advent of $\mathrm{CNC}$ machines and sophisticated computer algorithms it is now possible to manufacture and measure such lenses routinely. QED's MRF or Magneto-Rheological Finishing utilizes magnetically sensitive fluid with diamond or cerium oxide abrasive that serves as a polishing tool. First, a "spot" or removal function has to be determined. When the removal rate and spot shape are determined, the machine computes how long the lens has to dwell at a particular coordinate in order to achieve the desired correction. A delivery system scheme has been shown in Fig. 1.
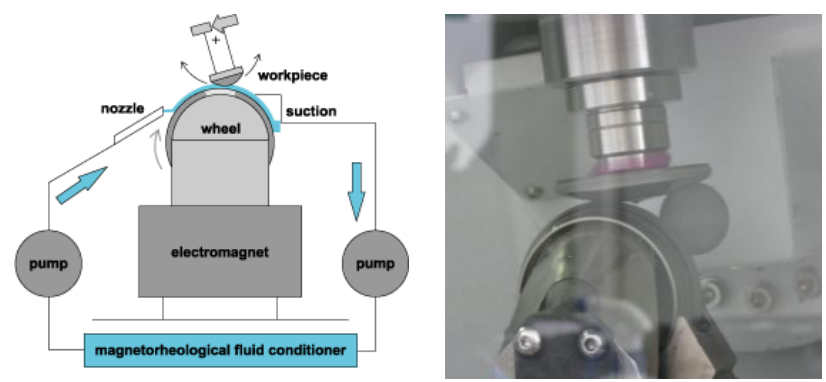

Fig. 1. Magneto-rheological fluid delivery system scheme and actual polishing run on Q22-XE machine.

${ }^{*}$ E-mail: sgogler@wat.edu.pl
Interferometric measurement of a spot has been shown in Fig. 2. Also the actual picture of a lens being polished has been shown in Fig. 1. As mentioned before, it is also important to be able to measure what one wants to manufacture. QED's Subaperture Stitching Interferometer (SSI-A) allows for aspheric shapes measurement with aspheric departure from the best fit sphere reaching over $100 \lambda$. The machine is a 6-axis CNC platform working

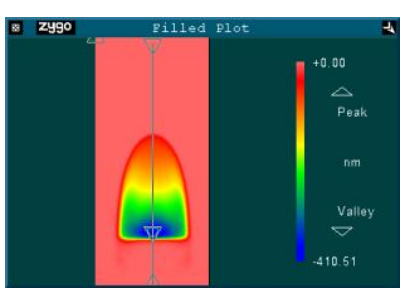

Fig. 2. Interferometric metrology of a typical MRF spot. together with a standard ZYGO interferometer. For an aspheric measurement, a lattice of subaperture measurements is created and after taking the set of measurements a state-ofthe-art algorithm connects subaperture interferograms into a full-field map. The machine in operation has been shown in Fig. 3. Also a screenshot showing measurement lattice and off-axis interferometric fringes have been shown.
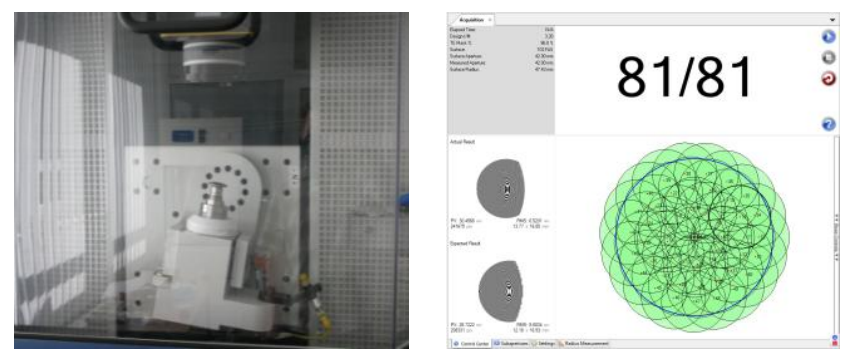

Fig. 3. QED SSI-A during operation (on the left) and measurement lattice of subapertures (on the right). On the left hand side of the lattice, measured and expected fringe patterns are shown.

Utilizing technologies describing the above aspheres of $100 \lambda$ of departure have been successfully manufactured in the Section of Infrared Technology and Thermovision of Institute of Optoelectronics in the Military University of Technology. Polishing of three elements will be described. Actual elements have been shown in Fig. 5. 


\begin{tabular}{|l|l|l|l|c|}
\hline $\begin{array}{l}\text { Element } \\
\text { no. }\end{array}$ & $\begin{array}{c}\text { Outer } \\
\text { diameter }\end{array}$ & $\begin{array}{c}\text { Clear } \\
\text { aperture }\end{array}$ & $\begin{array}{c}\text { Surface P- } \\
\text { V spec. }\end{array}$ & $\begin{array}{c}\text { Microrough- } \\
\text { ness spec. }\end{array}$ \\
\hline I & $13 \mathrm{~mm}$ & $11 \mathrm{~mm}$ & $<0.5 \mu \mathrm{m}$ & - \\
\hline II & $38 \mathrm{~mm}$ & $36 \mathrm{~mm}$ & $<0.5 \mu \mathrm{m}$ & - \\
\hline III & $75 \mathrm{~mm}$ & $67.5 \mathrm{~mm}$ & $<\lambda / 10$ & $<1 \mathrm{~nm}$ RMS \\
\hline
\end{tabular}

Tab. 1. Dimensions and specifications of polished parts.

Two elements are silicon lenses used in a MWIR thermal camera, the third one is a ZERODUR mirror substrate. Requirements for each element have been shown in Tab 1. Because of long run times, for the first two elements the polishing has been broken into several steps. Aspheric departure for these elements has been shown below. The first element (I) is a very strong asphere with $100 \lambda$ of departure and the polishing run has been split into three, the second element (II) with "only" $35 \lambda$ of departure has been split into two steps, as shown in Fig. 4. Polynomial coefficients for intermediate aspheres have been calculated by converting design prescription into Qbfs polynomial and dividing Qbfs coefficients by two or three as appropriate.
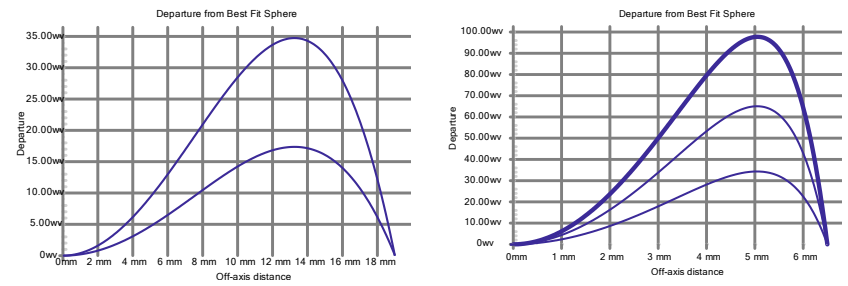

Fig. 4. Division of aspherization process into intermediate aspheres.

Description of the Forbes polynomials (Qbfs and Qcon) can be found in [1]. Additionally, on elements (I) and (II) the P-V surface error includes a vertex radius error, which is even a more demanding specification. Element (III) is a a parabolic mirror substrate with a significantly tighter surface specification, but the maximum aspheric departure is approximately $400 \mathrm{~nm}$.

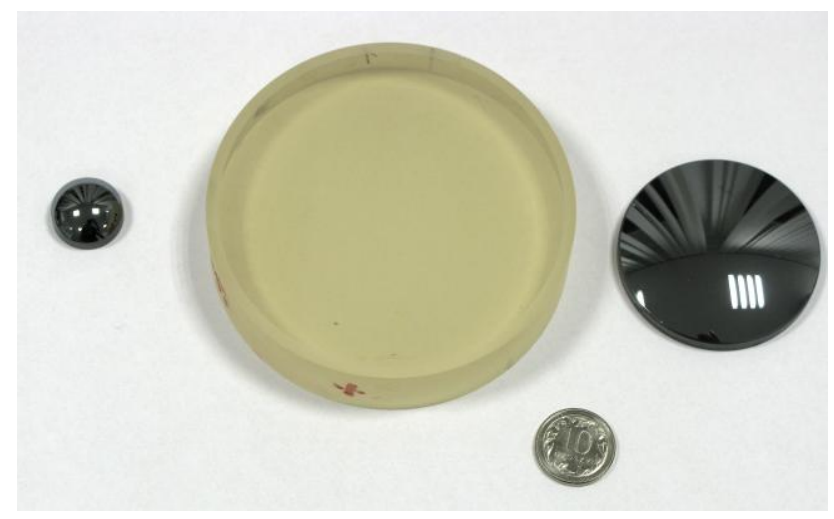

Fig. 5. Asherized elements (I), (III) and (II).

One problem with high volume removal using MRF is the mid-spatial frequency error. Once induced, it is very difficult to deal with. Since silicon elements work in the
MWIR spectral range, this is not a big concern. If a tight mid-spatial frequency specification (or PSD) is to be met the P-V amount of material to be removed should not exceed probably several micrometers. An example of MRF mid-spatial error has been shown in Fig. 6. The figure error after each polishing run has been presented. For the mirror substrate also a micro-roughness map has been shown.

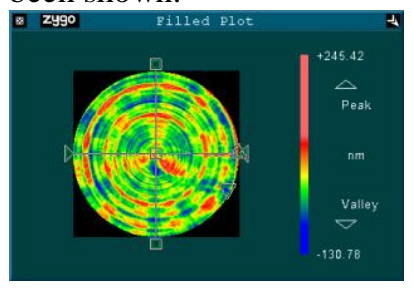

Fig. 6 MRF induced mid-spatial frequency error.
The MRF process routinely polishes surfaces to so-called "super polish" leaving micro-roughness of the surface (parameter $\mathrm{Ra}$ ) well below $1 \mathrm{~nm}$. It is also important to note, that the amount of material removed $(60 \mu \mathrm{m} \mathrm{P}-\mathrm{V})$ is almost extreme in the case of MRF technology and probably it is not the most economical approach. Ideally, most of the material should be removed with e.g. an asphere generator. The final polishing run is where MRF is best used. Nevertheless, this example shows how versatile the machine itself is.

In Figures 7, 8 and 9 below, subsequent steps of polishing of element (I) have been presented. Each measurement is taken against intermediate prescription. As can be seen, each step is approximately $8 \mu \mathrm{m}$ off the prescription. After reaching the final shape, several polishing runs were required to reach the final specification.
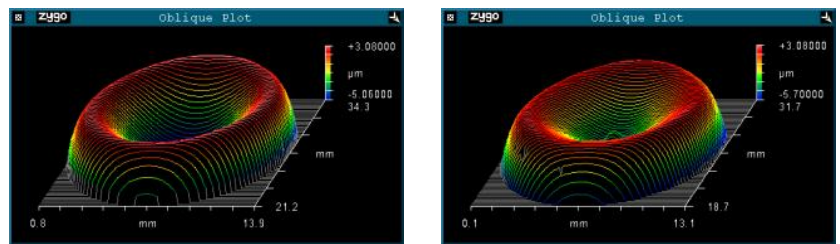

Fig. 7. Figure error from $1 / 3$ of the final prescription (on the left) and from $2 / 3$ on the right.
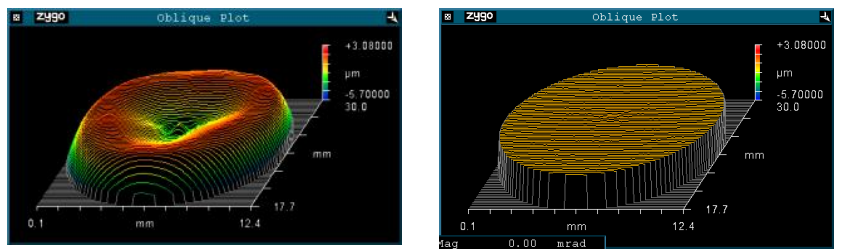

Fig. 8. Figure error of the final prescription, before correction runs.

After final correction, most of the remaining error is the center artifact. Since the P-V error is below $0.5 \mu \mathrm{m}$ and the amount of material removed was $60 \mu \mathrm{m}$, which makes less than $1 \%$ of error, as expected in such extreme cases. 


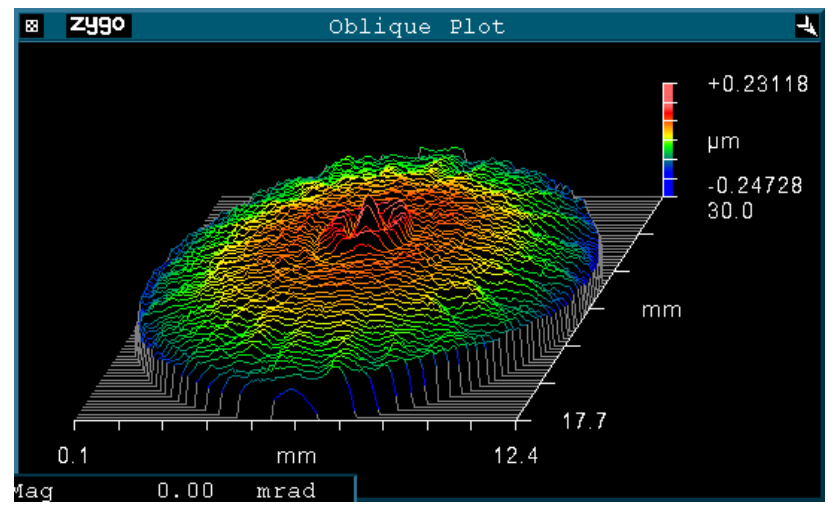

Fig. 9. Figure error of the final prescription and after final correction. Notice the scale change.

In Figures 10 and 11 are shown the results of polishing element (II). Again, after each step the surface is approximately $3 \mu \mathrm{m}$ off the intermediate prescription. Final correction runs are necessary to get the surface into specification.
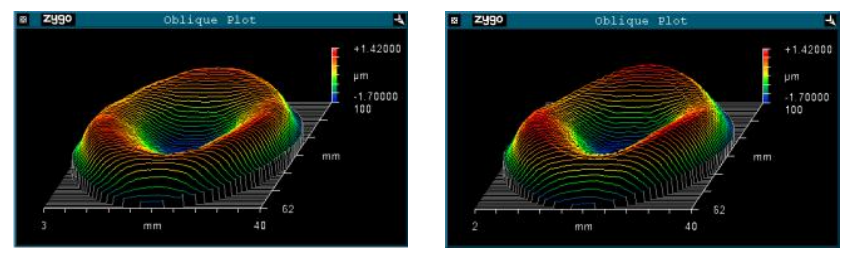

Fig. 10. Figure error from $1 / 2$ of the final prescription (on the left) and from the final prescription.

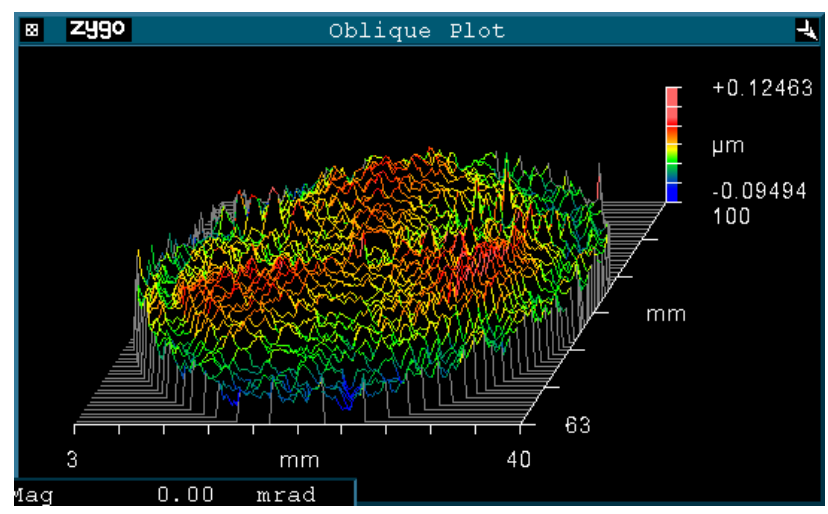

Fig. 11. Figure error of the final prescription after final correction. Notice the scale change.

Again, given the amount of material that has been taken off, this is a very good result, showing no center artifact, nor other artifacts typical for large volume removal with MRF (e.g. rings and spokes that can be seen in Fig. 6).

The last case is the ZERODUR parabolic mirror substrate. Even though specification on the surface accuracy was $\lambda / 10 \mathrm{P}-\mathrm{V}$ the surface has been brought to almost $\lambda / 50 \mathrm{P}-\mathrm{V}$ for the sake of demonstration purposes. Reaching the final accuracy of the surface figure required (aside from one shape correction) four correction runs. As can be seen in Figs. 9-11, the final figure error is approximately $\lambda / 50$, which is almost the edge of capabilities of the machine. At this point the limiting factor can be either accuracy or repeatability of metrology or both.
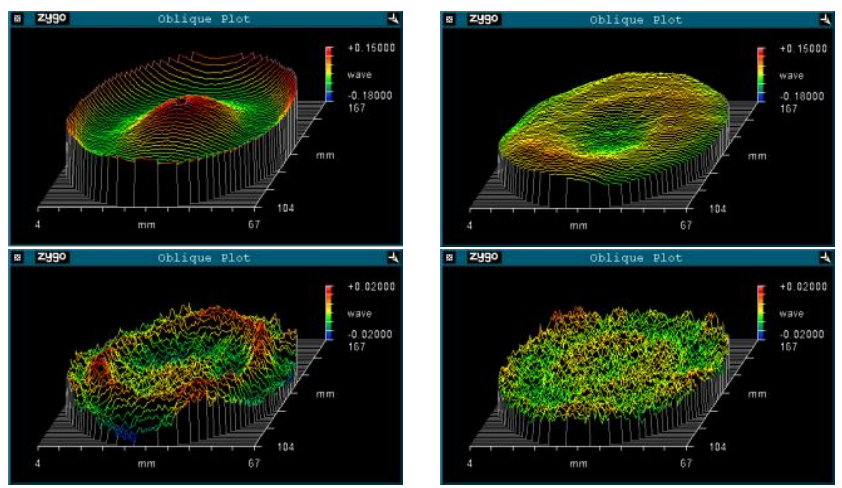

Fig. 12. Four correction results for the parabolic shape. Notice the scale change.

Even though the surface itself was already polished to a very low micro-roughness, after MRF polishing it improved from $\mathrm{Ra} 1.49 \mathrm{~nm}$ to $0.66 \mathrm{~nm}$ as shown below:
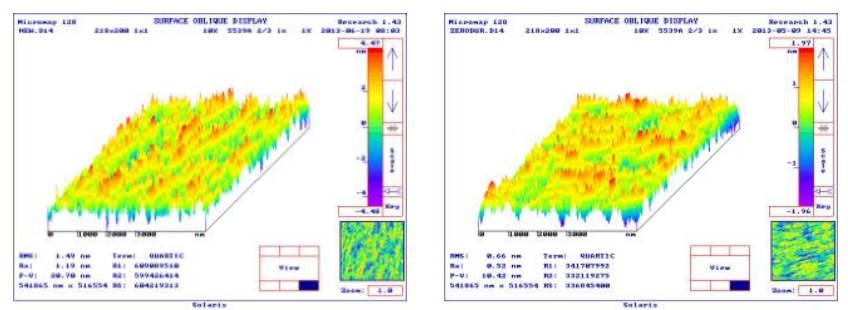

Fig. 13. Surface micro-roughness before (on the left) and after MRF polishing.

As has been shown, both QED's Q22-XE and SSI-A are capable of manufacturing super-precision aspheres as the ZERODUR parabola or extreme $100 \lambda$ aspheres. Accuracy of the final result depends on the amount of material that needs to be removed. It is also important to mention that $100 \lambda$ asphere of such a small dimension required over 50 hours of machine work, which suggests that it might not be the most efficient way to produce such an asphere. Nevertheless, the work has shown the feasibility of such a process as well as reaching $\lambda / 50$ accuracy.

\section{References}

[1] G.W. Forbes, Opt. Expr. 15(8), 5218 (2007). 